\title{
Grape Seed Proanthocyanidin Extract Moderated Retinal Pigment Epithelium Cellular Senescence Through NAMPT/SIRTI/NLRP3 Pathway
}

\author{
Wencui Wan ${ }^{1, *}$ \\ Wei Zhu $\mathbb{D}^{2, *}$ \\ Yan $\mathrm{Wu}^{3,4, *}$ \\ Yang Long (D)' \\ Hongzhuo Liu' \\ Weiwei Wan (1D) \\ Guangming Wan (D) \\ Jing $Y u \mathbb{D}^{3}$
}

'Department of Ophthalmology, The First Affiliated Hospital of Zhengzhou

University, Zhengzhou, People's Republic

of China; ${ }^{2}$ Department of Ophthalmology,

Changshu No. 2 People's Hospital,

Changshu, People's Republic of China;

${ }^{3}$ Department of Ophthalmology, Shanghai

Tenth People's Hospital, Tongji University,

Shanghai, People's Republic of China; ${ }^{4}$ Mois

Biotech Company, Shanghai, People's

Republic of China

*These authors contributed equally to this work
Correspondence: Guangming Wan Department of Ophthalmology, First Affiliated Hospital of Zhengzhou

University, I East Jianshe Road,

Zhengzhou, Henan, 450052, People's

Republic of China

Emailwgm6608@163.com

Jing $Y u$

No. 30I, Yanchang Road, Shanghai,

200072, People's Republic of China

Email dryujing@aliyun.com
Background: Retinal pigment epithelium (RPE) cellular senescence is an important process in degenerative retinal disorders. Grape seed proanthocyanidin extract (GSPE) alleviates senescence-related degenerative disorders; however, the potential effects of GSPE intake on RPE cellular senescence through regulating NAMPT/SIRT1/NLRP3 pathway remain unclear.

Methods: The effects of GSPE on NAMPT expression and NAD+ contents were detected with Western blot and assay kit in both in-vivo and in-vitro AMD models. Senescencerelated biomarkers, including p16, p21 expressions and $\beta$-gal staining, were conducted in different groups. The protective effects of GSPE treatment on the mitochondrial homeostasis and barrier function of RPE cells were detected using mtDNA lesions analyses, JC-1 staining, ZO1 staining and trans-epithelial cell resistance (TEER) detection. The expression of senescence-associated secretory phenotype (SASP) in different groups would be conducted with qPCR. To demonstrate the potential effects of NAMPT/SIRT1/NLRP3 pathway after GSPE treatment, the protein levels of relevant key regulators after applications of NAMPT inhibitor, Fk866, and SIRT1 inhibitor, EX-527.

Results: GSPE significantly improves the NAMPT expression and NAD+ content in aging mice, and thus alleviates the RPE cellular senescence. In advanced in-vitro studies, GSPE significantly up-regulated NAMPT content and thus relieved $\mathrm{H}_{2} \mathrm{O}_{2}$ induced NAD + depression through analyzing the NAD+ contents in different groups. In advanced analyses, it was reported that GSPE could alleviate mitochondrial permeability, mtDNA damage, ZO1 expression and SASP levels in aging RPE cells. Thus, GSPE treatment significantly decreased senescencerelated protein p16 and p21, as well as SASP levels in in-vitro aging model, and it was demonstrated that GSPE could illustrate a significant anti-aging effect. The Western blot data in GSPE treatment of aging RPE cells demonstrated that GSPE could significantly improve NAMPT and SIRT1 levels, and thus depressed NLRP3 expression.

Conclusion: This study indicated that GSPE alleviated RPE cellular senescence through NAMPT/SIRT1/NLRP3 pathway. This study highlighted the potential effects of GSPE on degenerative retinopathy through the crosstalk of NAD + metabolism, SIRT1 function and NLRP3 activation.

Keywords: cellular senescence, retinal pigment epithelium, nicotinamide phosphoribosyltransferase, sirtuin 1, inflammasome, NLRP3

\section{Introduction}

Cellular senescence, which is an important cause of physiological and pathological changes in the ocular structure including the retina, is responsible for the development of diabetic retinopathy (DR), retinitis pigmentosa (RP) and age-related 
macular degeneration (AMD). ${ }^{1}$ The senescence-related retinal degenerative disorders affect the visual functions of more than 500 million patients and even lead to blindness in severe cases. ${ }^{2}$ RPE is a polygonal cell layer located between the choroid and neuroretina. The dysfunction of RPE is regarded as a key factor leading to the occurrence of AMD, DR and RP. ${ }^{3,4}$ The senescence of RPE cells will impair its phagocytosis and barrier function, along with abnormal secretion of neurotrophic and angiogenic factors. ${ }^{5,6}$ Therefore, clarifying the mechanism of RPE cell senescence will help to deepen the understanding of the pathological mechanisms of retinal degenerative diseases, and thus provide clues for the new drugs development for retinal disease, which remains a major problem that needs to be solved urgently.

Nicotinamide adenine dinucleotide (NAD+), which is the core cofactor of mitochondrial function, plays a vital role in regulating cell metabolism and energy homeostasis. NAD + has been reported to be a potential target of the current anti-aging field. ${ }^{7,8}$ The decline of NAD+ content with age is closely related to the occurrence of age-related degenerative diseases; ${ }^{7,9}$ thus, NAD+ improvement would be a potential anti-aging therapy. In blue-light induced retinal damage (DR), as well as genetic glaucoma animal models, it is found that NAD + contents in the retina samples were significantly decreased, and exogenous NAD + supplementation could significantly prevent agerelated $\mathrm{NAD}+$ decrease and retinal dysfunction. ${ }^{10,11}$ A recent study analyzed the metabolic pathways in the RPE samples of AMD patients and it was found that $\mathrm{NAD}+$ is decreased significantly. Nicotinamide phosphoribosyltransferase (NAMPT), also known as visfatin and pre-B cell enhancing factor (PBEF), is a rate-limiting enzyme that catalyzes the synthesis of nicotinamide into nicotinamide mononucleotide (NMN). NMN is the direct precursor of $\mathrm{NAD}+$ in the NAD salvage synthesis pathway and could be transformed into NMN directly. ${ }^{12}$ In retinal degenerative diseases, the expression of NAMPT and the $\mathrm{NAD}+$ content is consistent and reduced compared with the healthy controls. Therefore, we conjecture that abnormal NAD + metabolism is involved in RPE cellular senescence, and thus the key regulatory factors of NAD+ metabolic pathway, NAMPT, is related to the development of retinal degenerative disorders.

Grape seed proanthocyanidin extract (GSPE) is extracted from whole grape seeds and regarded as an important source of polyphenolic bioflavonoid. ${ }^{13,14}$ As reported in the previous studies, GSPE exhibits antioxidant, anti-inflammatory, and anti-aging properties. $^{15,16}$ GSPE could up-regulate the NAMPT expression, ${ }^{17}$ however, the potential protective role of GSPE induced NAMPT expressions in the RPE cellular senescence has not been studied. Therefore, based on these mentioned viewpoints, we hypothesize that GSPE treatment could inhibit the RPE senescence through upregulation of NAMPT. Advanced in-vivo and in-vitro experiments would be conducted to detect the effects of GSPE on the RPE cell function as well as the potential pathological mechanisms through NAMPT/SIRT1/NLRP3 pathway.

\section{Materials and Methods Reagents}

The GSPE with over $98 \%$ proanthocyanindin purity was purchased from Rongsheng Biotechnology Co., Ltd. (Cat No.: 84929-27-1; Xi'an, China) and dissolved in drinking pure water for in-vivo study and in DMSO (Cat No.: C6164, Signa, USA) for in-vitro treatment. NAMPT inhibitor, Fk866 (Cat No.: F8557), and SIRT1 inhibitor, EX527 (Cat No.: E7034), were purchased from Sigma (Sigma-Aldrich, USA). The NAD+ precursor, NMN with a purity of over $99.5 \%$ was purchased from Hygieia Biotechnology Co., Ltd (Cat No.: XJY01210128, Shenzhen, China).

\section{Animals}

All animal experiments were conducted in accordance with the Association for Research in Vision and Ophthalmology Statement for use of Animals in Ophthalmic and Vision Research. The animal protocol was approved by the Animal Ethics Committee of Zhengzhou University. Both male and female $\mathrm{C} 57 \mathrm{BL} / 6 \mathrm{~J}$ mice of different age groups were obtained from a commercial vendor (Sipuer-Bike Laboratory, Shanghai). The mice would be housed under a pathogen-free environment with a 12:12 h light/dark cycle and free access to laboratory chow and water. Animal rooms were maintained at $20-22^{\circ} \mathrm{C}$ with $30 \%-70 \%$ relative humidity. Peripheral blood samples were collected and plasma was isolated and stored in $-80^{\circ} \mathrm{C}$ until use. Besides, ocular samples were collected and then RPE was isolated gently from the choroidal layer in accordance with the previous study in different time points. ${ }^{18}$ The obtained animal samples were stored in $-80^{\circ} \mathrm{C}$ until advanced experiments. GSPE treatment would be conducted through adding GSPE to drinking water and GSPE treatment conducted in 
the 15 months aged mice. After three months of GSPE treatment, the 18 months aged mice would be used in the following experiments.

\section{Cell Culture}

The ARPE19 cells were purchased from American-type culture collection (Cat No.: CRL-2302, ATCC, USA) and cultured with Dulbecco's modified Eagle medium/F12 (DMEM/F12) (Cat No.: SH30023.01B, HyClone, USA) supplemented with $10 \%$ heated-inactivated fetal bovine serum (FBS, Cat No.: 10099-141, Gibco, USA), 100 U/ $\mathrm{mL}$ penicillin, and $100 \mu \mathrm{g} / \mathrm{mL}$ streptomycin (Cat No.: 15140-122, Gibco, USA). The ARPE-19 cells would be maintained at $37^{\circ} \mathrm{C}$ in a humidified chamber with $5 \% \mathrm{CO}_{2}$ and 1:3 subcultures were conducted using trypsin-EDTA solution (Cat No.: 15140-122, Gibco, USA).

\section{RNA Extraction and Real-Time PCR}

Total RNA from both animal examples and cultured cells were extracted with Trizol reagent (Cat No.: 15596026, Invitrogen, USA) following the manufacturer's protocol. cDNA synthesis was conducted using Transcriptor First Strand cDNA Synthesis Kit (Cat No.: 04896866001, Roche, USA). RT-PCR was performed with Applied Biosystem $^{\text {TM }}$ SYBR ${ }^{\mathrm{TM}}$ using the TaqMan Multiplex RealTime PCR Solution (Cat No.: 4367659, Thermo, USA) according to the manufacturer's instructions. Relative mRNA expressions would be calculated with the ${ }^{\Delta \Delta} \mathrm{Ct}$ method. The primers used in this current study were listed in Supplemental Table 1. Three independent experiments were conducted for each sample and relevant results were used in advanced analyses.

\section{NAD+ Content Assay}

The NAD+ contents in both animal samples and cellular homogenates were detected using a commercial NAD+ assay kit (Cat No.: MAK037, Sigma-Aldrich, USA). All the experiments were conducted according to the manufacturer's instructions.

\section{In-vitro Aging RPE Model}

The in-vitro aging model of RPE cells was generated according to a protocol previously described in our lab ${ }^{19}$ with slight modifications. In general, the APRE-19 cells were placed in petri dishes, and then exposed to 800 $\mu \mathrm{M} \mathrm{H} \mathrm{H}_{2} \mathrm{O}_{2}$ for 2 hours at $37^{\circ} \mathrm{C}$ for five consecutive days. After that, the medium would be replaced with Fresh complete media after $\mathrm{H}_{2} \mathrm{O}_{2}$ treatment. The aging RPE cells would be collected in the other day after the last $\mathrm{H}_{2} \mathrm{O}_{2}$ treatment and the aging in-vitro model would be used in the following studies.

\section{Cell Viability}

The cell viability was detected in this study with MTT assay. MTT dye (Cat No.: M1025, Solarbio, China) was added to the 96-well plate with ARPE-19 cells in different groups and incubated for four hours at $37^{\circ} \mathrm{C}$. After replacing the culture media with $150 \mu \mathrm{L}$ of DMSO to each well, the 96-cell plates were shaken thoroughly until the crystals dissolved. Absorbance was measured at a wavelength of $490 \mathrm{~nm}$ using the Thermo Scientific Fluorescent microplate reader (Thermo Fisher Scientific, USA). Three independent experiments were conducted for each group and data were used for advanced analyses.

\section{Senescent $\beta$-Galactosidase Assay}

Cellular senescence marker, $\beta$-galactosidase ( $\beta$-Gal) activity, was used in the measurement of senescent status in different cell models, and the assay was performed with a commercial assay kit (Cat No.: C0602: Beyotime Biotechnology, China) according to the manufacturer's protocols. The view and counting would be conducted under the brightfield of the Leica Microscope (Leica Biosystems, Germany). The senescent cells expressing SA $\beta$-gal were positive cells and stained blue. Positive cell rates were recorded in each group and the data would be used in advanced analyses.

\section{mtDNA Lesions Measurement}

As NAD+ was an important contributor to DNA damage repairment, the mtDNA lesion measurement was conducted in each group. The detection of mtDNA was performed using a well-validated QPCR-based detection assay, as previously described. ${ }^{20}$ The DNA extraction was conducted using a genomic-tip kit (Cat No.: 10043, Qiagen, CA) and the extracted DNA would be stored in $-80^{\circ} \mathrm{C}$ before experiments. The principle of mtDNA lesion measurement was that any lesion on a DNA template would block the function of thermostable polymerase and therefore only the DNA templates without any DNA damages could be amplified. After DNA templates quality control, the qPCR would be conducted with a High-Stability PCR Kit (Cat No.: E00043, GenScript, China). The $\beta$-globin product was used as an inner-control for the detection of relative 
mtDNA lesion as described previously. Three independent experiments were conducted with three repetitions.

\section{Mitochondrial Membrane Potential}

The JC-1 dye accumulated in the mitochondria in a potential-dependent manner and thus could be used to detect the membrane potential of cells, tissues or purified mitochondria. JC-1 was an ideal fluorescent probe widely used to detect the $\Delta \Psi \mathrm{m}$ position of mitochondrial membrane (Cat No.: C2006, Beyotime Biotechnology, China). After aspirating culture media in the 6-well plate and washing the cells with PBS, $1 \mathrm{~mL}$ of JC-1 dye working solution was added into each well. After incubating the mixed working system for 20 minutes at $37^{\circ} \mathrm{C}$, the cells would be washed with the JC-1 Buffer $(1 \times)$ and placed on ice. The cells would be observed under a confocal microscope (Leica, Germany). The polymer emits strong red fluorescence $(E x=585 \mathrm{~nm}$, $\mathrm{Em}=590 \mathrm{~nm}$ ) and the monomers produced green fluorescence $(E x=514 \mathrm{~nm}, E m=529 \mathrm{~nm})$. The fluorescence rate of monomers/polymer was detected and analyzed.

\section{Trans-Epithelial Cell Resistance Measurement}

Trans-epithelial cell resistance (TEER) was conducted to identify the cellular permeability with TEER24 (Applied Biophysics, USA). ARPE-19 cells were inoculated on the bottom membrane of the upper layer of the transwell chamber $\left(0.3 \times 10^{4} /\right.$ well $)$, and $1.2 \mathrm{~mL}$ of the complete cell culture medium was added to the chamber. The TEER was measured after the cell was confluent. After debugging the TEER24 epithelial volt ohmmeter and wiping the electrode pads with alcohol, the electrode sheets were immersed into the inner and outer culture medium, respectively. The resistance of the blank control group (without any cells inoculated) and the experimental group was measured vertically in sequence. The measurement was repeated three times for each hole, and three multiple holes were set for each group. Calculation of TEER value was conducted as following: TEER= (resistance value experimental group-resistance blank control) $\times 0.3$ and the unit was $\Omega / \mathrm{cm}^{2}$.

\section{Cellular Immunofluorescence}

After plating the ARPE-19 cells onto six-well plates and generating cells grown on a coverslip, the coverslip was washed once with a PBS buffer, and then the cells would be fixed for $20 \mathrm{~min}$ at room temperature. To break the membrane, $0.2 \%$ Triton $\mathrm{X}-100$ was added following with three times washes with PBS. Incubation with blocking solution (PBS containing 10\% goat serum) was conducted for $2 \mathrm{~h}$ at $4{ }^{\circ} \mathrm{C}$ and then the cells would be incubated with dilute primary antibody (anti-ZO1, Cat No.: ab221547, Abcam, UK; anti-NLRP3, Cat No.: ab263899, Abcam, UK) for immunostaining in a cold room overnight. The cells were washed three times with PBS and then the secondary antibody was added to incubate with the cells for $1 \mathrm{~h}$ in the dark. Drop the DAPI \& anti-quencher mixture onto the glass slide and flip the cover glass. Observation and record of the immunofluorescence photographs were conducted with a Zeiss confocal microscope (Leica Biosystems, Germany) under dark.

\section{Western Blot}

After extracting the total protein from tissues and cellular samples using RIPA buffer with protease inhibitors, BCA quantitative measurements of protein concentrations were conducted. The relative expressions of the target proteins were normalized to $\beta$-actin. A total of $20 \mu \mathrm{g}$ protein for each sample was separated by SDS-PAGE and transferred onto PVDF membrane (Cat No.: IEVH00005, Millipore, USA). After incubating with the milk in TBS for blocking, the membrane would be incubated the corresponding primary antibody, including anti-p16 ${ }^{\mathrm{INK} 4 \mathrm{a}}$ (Cat No.: ab108349, Abcam, UK), p21 $1^{\text {Waf/Cip1 }}$ (Cat No.: ab109520, Abcam, UK), SIRT1 (Cat No.: 2310, Cell Signaling, USA), NLRP3 (Cat No.: ab263899, Abcam, UK), apoptosis-associated speck-like protein (ASC, Cat No.: 13833, Cell Signaling, USA), pro-caspase-1 (Cat No.: ab179515, Abcam, UK), caspase 1 (Cat No.: ab207802, Abcam, UK) and $\beta$-actin (Cat No.: sc-47724, Santa Cruz, USA), at $4^{\circ} \mathrm{C}$ overnight. On the second day, the membrane was washed three times with TBST and incubated with the secondary antibody (Cat No.: sc-2357 and sc-2005, Santa Cruz, USA) for $1 \mathrm{~h}$. The density of the bands was quantified using Labworks image acquisition software (UVP, USA) and was quantized using ImageJ software (NIH, USA).

\section{ELISA Assays}

The production of IL-1 $\beta$ and IL18 of ARPE-19 cells in different groups was measured using IL-1 $\beta$ (Cat No.: PI305, BioPioneer Tech, China) and IL-18 (Cat No.: PI558, BioPioneer Tech, China) ELISA kit according to 
the manufacturer's instructions. Samples were quantified with a corrected value of 450 and $570 \mathrm{~nm}$ using the absorbance measurements in the Thermo Scientific Fluorescein microplate reader (Thermo Fisher Scientific, USA). Three replications for all the samples were adopted and the data of three independent studies were used in the final analyses.

\section{Statistical Analysis}

In this study, the data were presented as a mean \pm standard deviation (SD). The non-paired $t$ test would be used for the difference detection non-paired $t$ test, and one-way analysis of variance (ANOVA) followed by the Newman-Keuls method for multiple comparison analysis test among over two groups was used for analyses. The $P$ value $<0.05$ denotes statistical significance.

\section{Results}

\section{Decreased NAMPT Expression and NAD + Levels in RPE and Plasma Samples Could Be Rescued by GSPE Supplement in Aging Mice}

$\mathrm{NAD}+$ metabolism was a key regulator in the increasing of aging and salvage pathway provided about $85 \%$ of total NAD + . As described in Figure $1 \mathrm{~A}, \mathrm{NAD}+$ was transformed into NAM through NAD + consume. Then, the NAM would be transformed into NMN after being catalyzed by NAMPT. NMN would then be used in NAD+ synthesis by NMNAT1-3 enzyme and thus the NAD+ salvage metabolism cycle was completed. Compared with the young mice ( 2 months aged), lower NAMPT expression in 18-month aged mice and decreased NAD + contents in 12- and 18-months aged mice were detected $(P<0.05)$. After supplementing $250 \mathrm{mg} / \mathrm{kg}$ GSPE from 15 to 18 months age, a significant increased NAMPT expression and improved NAD + content was detected $(P<0.05$, Figure $1 \mathrm{~B}$ and $\mathrm{C}$ ). A similar expression pattern was observed in the mouse plasma and NAMPT/NAD+ levels decline was observed in the aging mice. The intake of $250 \mathrm{mg} / \mathrm{kg}$ GSPE for three months significantly improved the NAMTP expression and NAD + content in plasma $(P<0.05$, Figure 1D and $\mathrm{E})$.

\section{GSPE Alleviated Aging Related Cellular Senescence and SASP in Mouse RPE}

To detect the protective effects of GSPE supplement in the aging mice, the expressions of cellular senescence-related markers, $\mathrm{p} 16^{\mathrm{INK} 4 \mathrm{a}}$ and $\mathrm{p} 21^{\mathrm{Waf} / \mathrm{Cip} 1}$, as well as the SASP contents were reported. As showed in Figure 2A, p16 ${ }^{\mathrm{INK} 4 \mathrm{a}}$ and $\mathrm{p} 21^{\mathrm{Waf} / \mathrm{Cip} 1}$ expressions were increased in the aging mice (18 months aged) and $250 \mathrm{mg} / \mathrm{kg}$ GSPE would decline these cellular senescence-related biomarkers. As SASP involved a series of pro-inflammatory factors, chemokines as well as proteases. Common SASP related biomarkers, including tumor necrosis factor- $\alpha$ (TNF- $\alpha$ ), transforming growth factor- $\beta 1$ (TGF- $\beta 1$ ), interleukin- $1 \beta$ (IL1 $\beta$ ), interleukin-6 (IL6), interleukin-8 (IL8), interleukin-18 (IL18), monocyte chemotactic protein 1 (MCP-1), granulocyte-colony stimulating factor (G-CSF), chemokine (C-X-C motif) ligand 1 protein (CXCL1), chemokine (C-X-C motif) ligand 2 protein (CXCL2), inositol requiring enzyme1 $\alpha$ (Ire1 $\alpha)$, plasminogen activator inhibitor-1 (PAI1), matrix metalloproteinases-1 (MMP-1), matrix metalloproteinases-3 (MMP-3) and tissue inhibitor of metalloproteinase-2 (TIMP-2) were detected. The differently expressed IL18, TGF- $\beta 1$, IL1 $\beta$, TIMP-2, MMP-3, IL8 and MCP-1 in the RPE tissues of the aging mice were detected $(P<0.05)$. Most significantly regulated SASP were reserved by three months of GSPE supplementation and the visualized data as well as the detailed $P$ values are presented in Figure 2B.

\section{GSPE Extenuated RPE Senescence in the in-vitro Model}

To detect the effects of GSPE treatment in the normal and aging ARPE-19 cells, GSPE in different concentrations (0.1, $1,10,50$ and $100 \mu \mathrm{g} / \mathrm{mL}$ ) were applied in the in-vitro model. As showed in Figure 3A and B, GSPE failed to affect the cell viability of the normal ARPE-19 cells; however, 10 and more than $10 \mu \mathrm{g} / \mathrm{mL}$ of GSPE significantly improved the cell viability in the aging RPE cells. When the NAD+ contents and NAMPT expressions in the aging ARPE-19 cells were considered, a significant deceased NAD + and NAMPT contents were observed in the aging RPE cells and these two NAD+ metabolism markers were upregulated with $10 \mu \mathrm{g} / \mathrm{mL}$ or higher concentrations of GSPE treatments $(P<0.001$, Figure $3 \mathrm{C}$ and $\mathrm{D})$. In advanced $\beta$-gal activity detection, it was found that $\beta$-gal activity was significantly increased in the aging RPE cells and the activity was alleviated by $10 \mu \mathrm{g} / \mathrm{mL}$ or higher concentrations of GSPE treatment $(P<0.001$, Figure $3 \mathrm{E})$. The effects of GSPE on the cell viability, NAD + metabolism and $\beta$-gal activity were consistent and the concentration of $10 \mu \mathrm{g} / \mathrm{mL}$ would be used in the following experiments. 
A

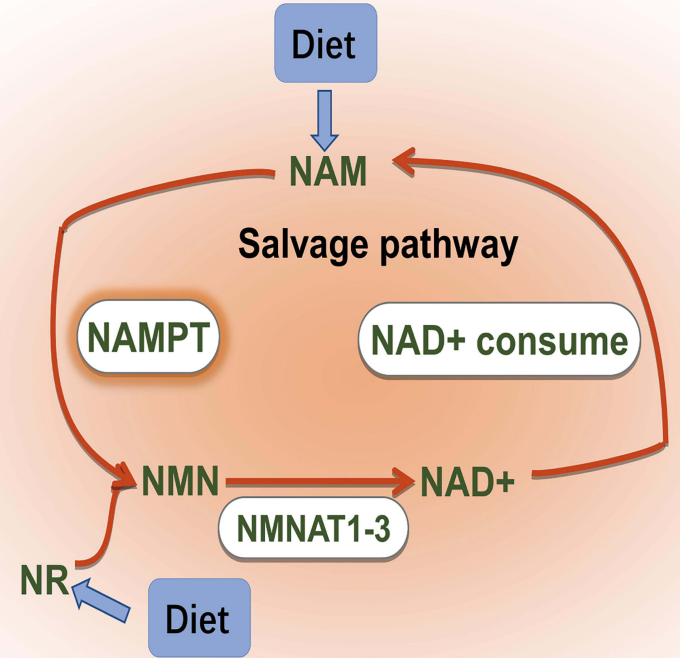

B

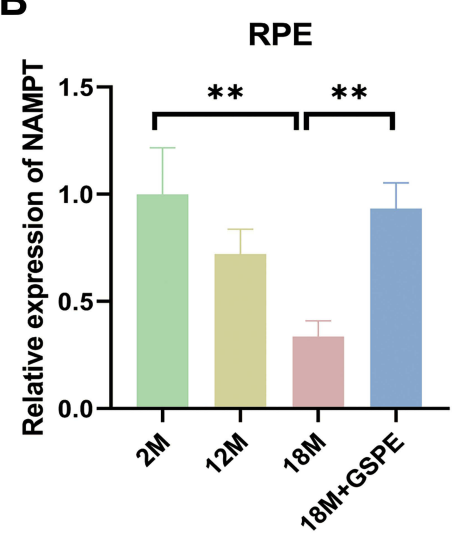

C RPE

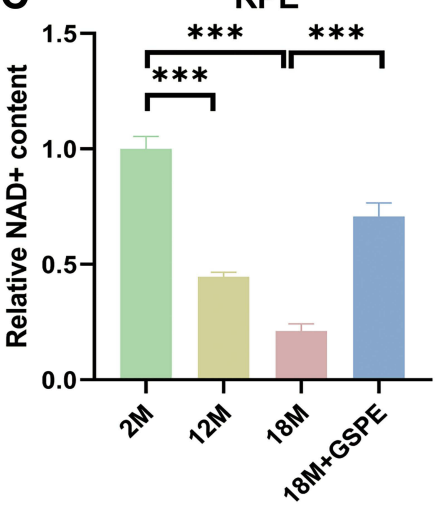

D

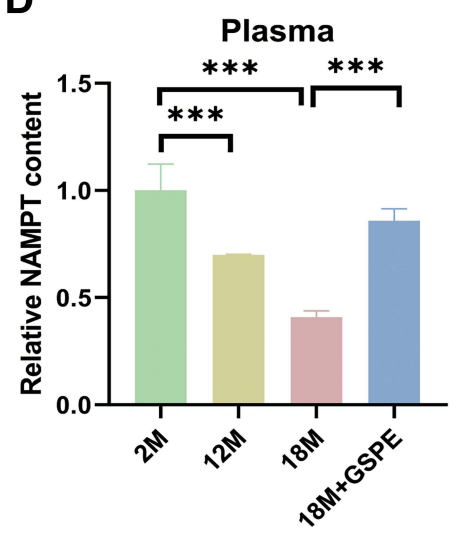

E

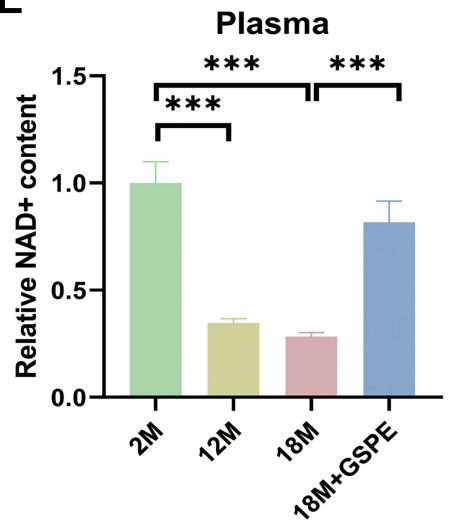

Figure I Decreased NAMPT and NAD+ levels in RPE and plasma could be improved by GSPE treatment in aging mouse. (A) The salvage pathway of NAD+ metabolism: NAD+ was transformed into NAM through NAD+ consume, the NAM would become NMN after being catalyzed by NAMPT and NMN would also be transformed into NAD+ by NMNATI-3 enzyme. (B-C) Compared with 2-month aged mice, NAMPT expression is reduced in 18-month aged mice, and NAD+ content is reduced in 12month and 18-month aged mice. After supplementing with GSPE at a concentration of $250 \mathrm{mg} / \mathrm{kg}$ at 15 to 18 months of age, significant increase in NAMPT expression and NAD+ content. $n=3$, **: $P<0.0$ I. (D-E) The decrease of plasma NAMPT/NAD + level was related to the increased mouse age. Ingestion of $250 \mathrm{mg} / \mathrm{kg}$ GSPE for three months can significantly improve the content of NAMTP and NAD+ in plasma. $n=3, * *: P<0.01, * * *: P<0.001$. 


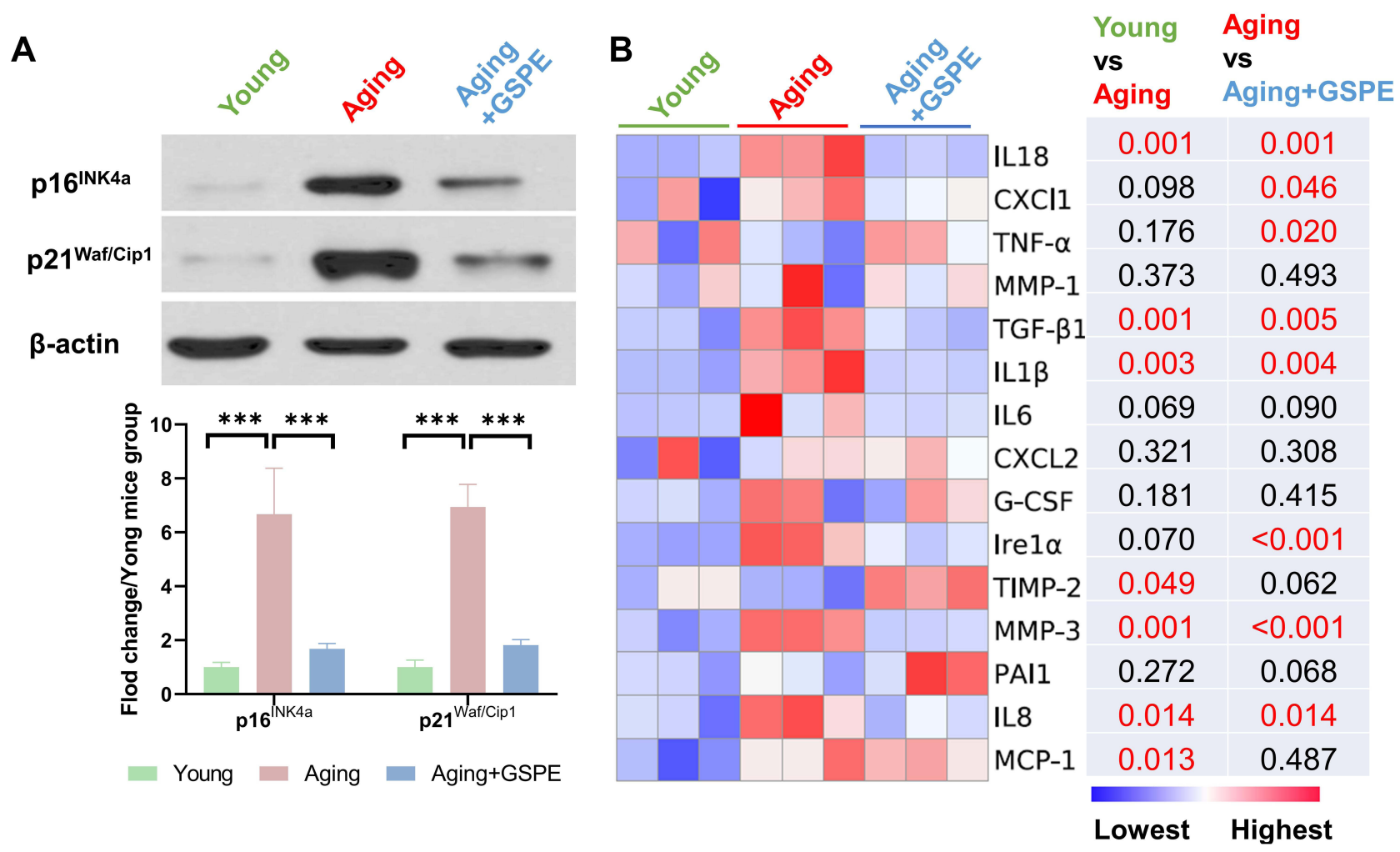

Figure 2 GSPE alleviated aging related cellular senescence and SASP in mouse RPE. (A) $p \mid 6^{\mid N K 4 a}$ and $p 2 I^{\text {Waf/Cipl }}$ expressions were increased in the aging mice (I8 months aged) and the GSPE in $250 \mathrm{mg} / \mathrm{kg}$ would decline these cellular senescence biomarkers. The representative western-blot gels and statistical results were presented. $\mathrm{n}=3$, $* * *$ : $P<0.00 I$. (B) The SASP factors in the young, aging and aging mice treated with three-month GSPE supplementation. The expressions of TNF- $\alpha$, TGF- $\beta$ I, ILI $\beta$, IL6, IL8, ILI8, MCP-I, G-CSF, CXCII, CXCL2, IreI $\alpha$, PAII, MMP-I and MMP-3 were visualized by s heatmap. The lowest expression was marked as blue and the highest expression was marked as red. The $P$ values of comparison of young vs aging as well as aging vs aging mice treated with GSPE were presented and any $P$ values of significant differences were marked red $(P<0.05)$. $n=3$.

\section{GSPE Alleviated Mitochondrial Homeostasis, Barrier Function and SASP Levels of Aging RPE Cells}

As NAD+ metabolism was closely related to mitochondrial homeostasis, the mtDNA lesions were detected with a classical PCR-based method. Besides, the mitochondrial membrane permeability was analyzed with JC-1 staining. As showed in Figure 4A, GSPE treatment significantly alleviated the mtDNA lesions, which was significantly increased in the aging RPE cells $(P<0.001)$. Increased mitochondrial membrane permeability was related to the worsening mitochondrial function and it would demonstrate more monomers JC1 staining in the mitochondria. After GSPE treatment, the damaged mitochondrial membrane permeability was significantly improved $(P<0.001$, Figure 4B). Considering that the physiological functions of RPE cells included barrier function and growth factor secretions, the effects of GSPE treatment on the barrier function and SASP on aging RPE cells were reported in this study. Both the cellular barrier marker (ZO1) and barrier function (TEER) were detected in this study. It was reported that GSPE could significantly improve the expression of $\mathrm{ZO} 1$ and then reinstate the TEER in the aging RPE cells $(P<0.05$, Figure $4 \mathrm{C}$ and D). The abnormal growth factor secretions of RPE cells were related to the incidence of retinal diseases and SASP related factors were reported to be significantly improved in the RPE cells of the aging mice. In this study, significantly expressed TGF- $\beta 1$, IL1 $\beta$, IL6, IL8, MCP-1, G-CSF, CXCL2, MMP3 and TIMP-2 were detected $(P<0.05$, Figure 4E). Compared with aging RPE cells, GSPE treatment could significantly alleviate the expression of the most dysregulated SASP factors $(P<0.05)$.

\section{GSPE Regulated NAMPT/SIRTI Pathway in Aging RPE Cells}

As demonstrated in Figure 5A, the protective effects of GSPE on cellular senescence in aging RPE cells were blocked by NAMPT inhibitor, Fk866, while the supplementation of NMN significantly reinstated the protective effect. Advanced study on the NAD+ status demonstrated 
A

Normal

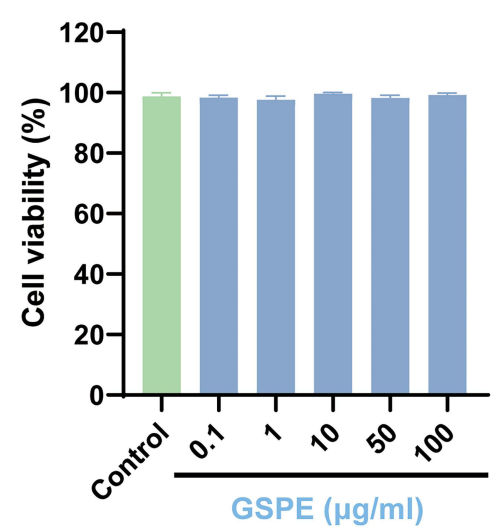

D

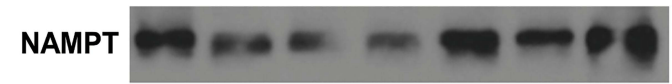

$\beta$-actin
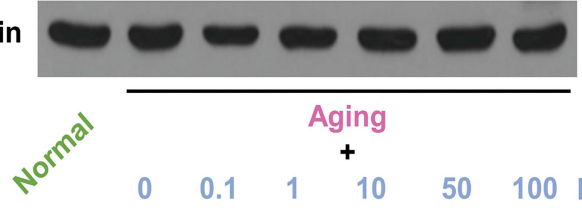

50

$100 \mu \mathrm{g} / \mathrm{ml}$ GSPE

$\mathbf{E}$

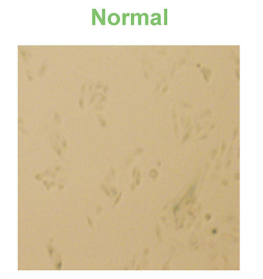

Aging $+1 \mu \mathrm{g} / \mathrm{ml}$ GSPE

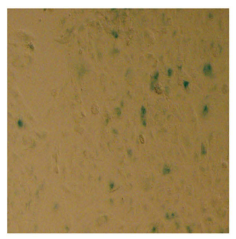

Aging $+100 \mu \mathrm{g} / \mathrm{ml}$ GSPE

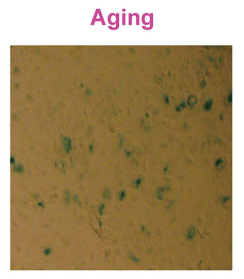

Aging $+10 \mu \mathrm{g} / \mathrm{ml}$ GSPE
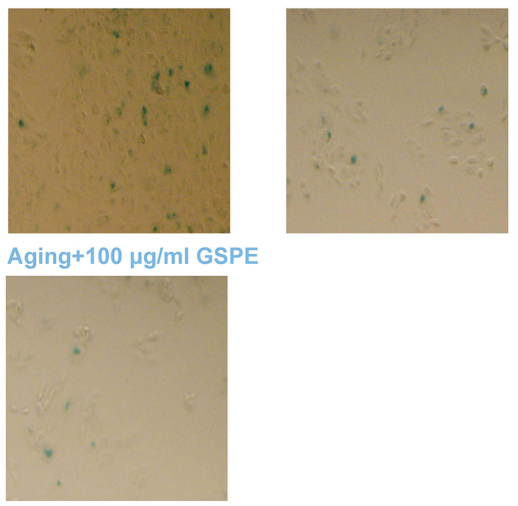
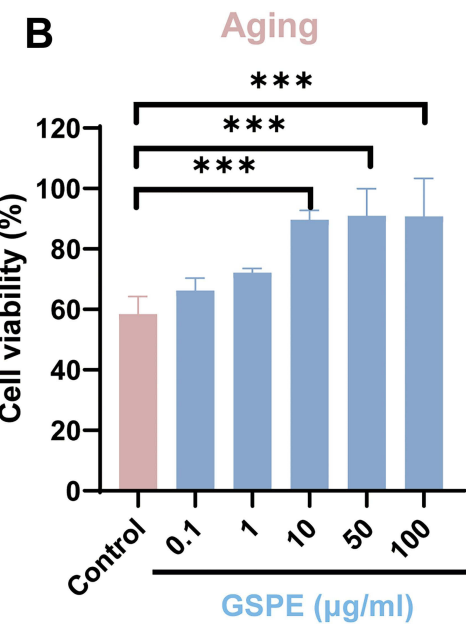

Aging $+0.1 \mu \mathrm{g} / \mathrm{ml} \mathrm{GSPE}$

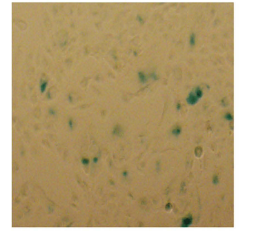

Aging $+50 \mu \mathrm{g} / \mathrm{ml}$ GSPE

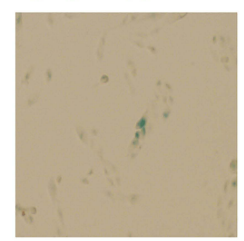

C

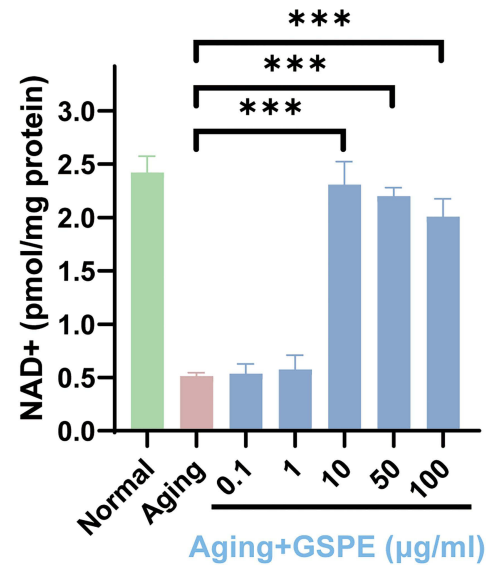

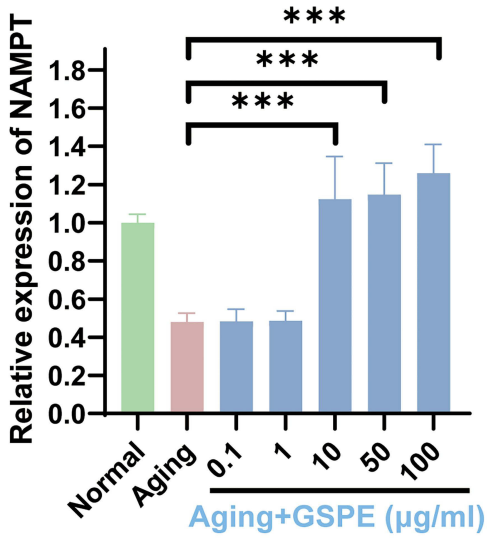

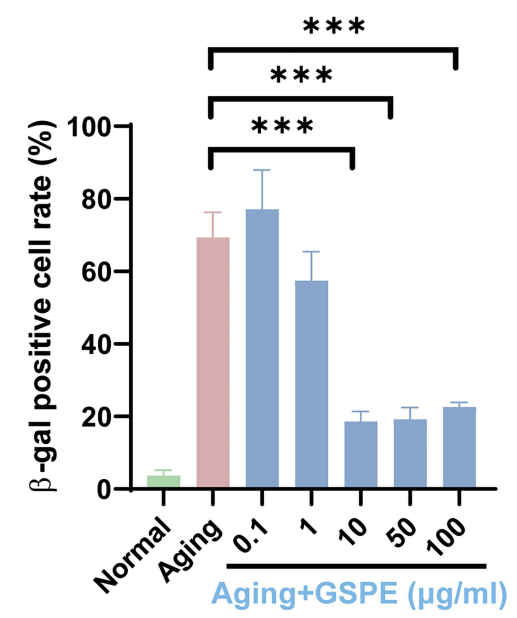

Figure 3 GSPE extenuated RPE cellular senescence of the in-vitro model. (A-B) GSPE failed to affect the cell viability of normal ARPE-19 cells, while GSPE at I0 and I0 $\mu$ / $m L$ significantly improved the cell viability of senescent RPE cells. $n=3$, ***: $P<0.001$. (C) NAD + content was decreased significantly in senescent RPE cells, and the NAD + content was up-regulated with $10 \mu \mathrm{g} / \mathrm{mL}$ or higher concentrations of GSPE treatments. $\mathrm{n}=3, * * *$ : $P<0.00 \mathrm{I}$. (D) The expression of NAMPT was decreased significantly in senescent RPE cells, and it was up-regulated with $10 \mu \mathrm{g} / \mathrm{mL}$ or higher concentrations of GSPE treatments. The representative western-blot gels and statistical results were presented. $n=3$, ***: $P<0.00 I$. (E) The $\beta$-gal activity is significantly increased in senescent RPE cells, and treatment with GSPE at a concentration of I0 $\mu$ g/mL or higher reduces the activity. $\mathrm{n}=3$, ***: $P<0.00 \mathrm{I}$. 
A

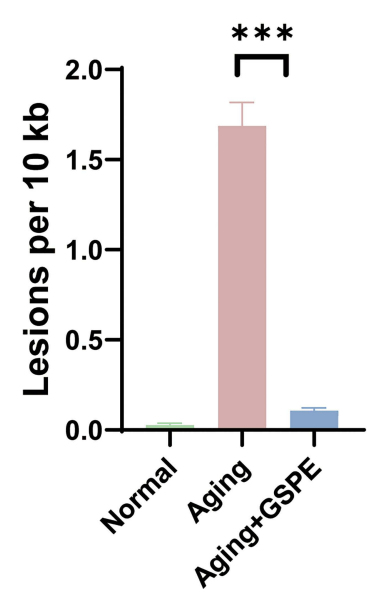

C
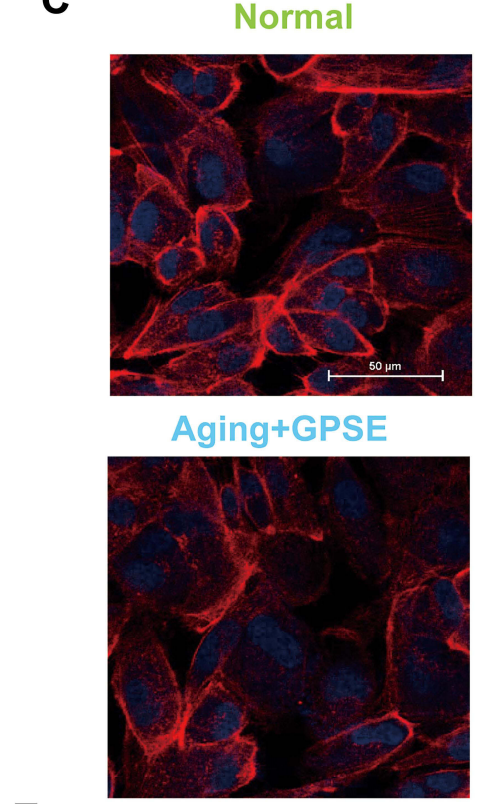

E

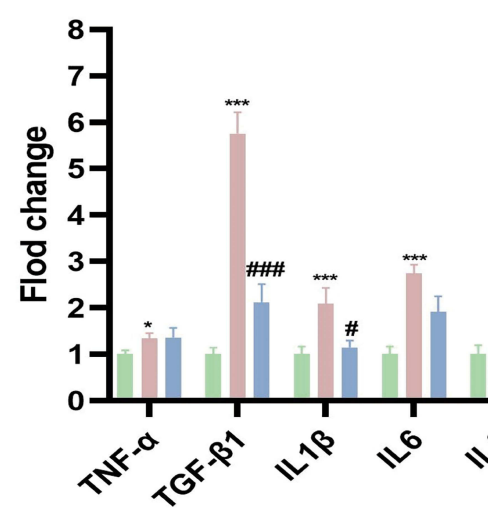

B

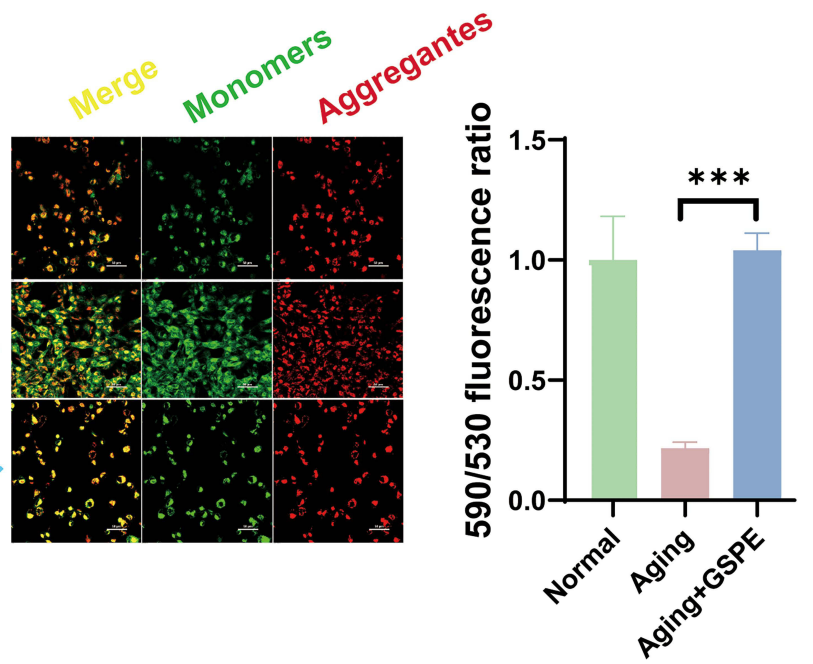

D

Aging
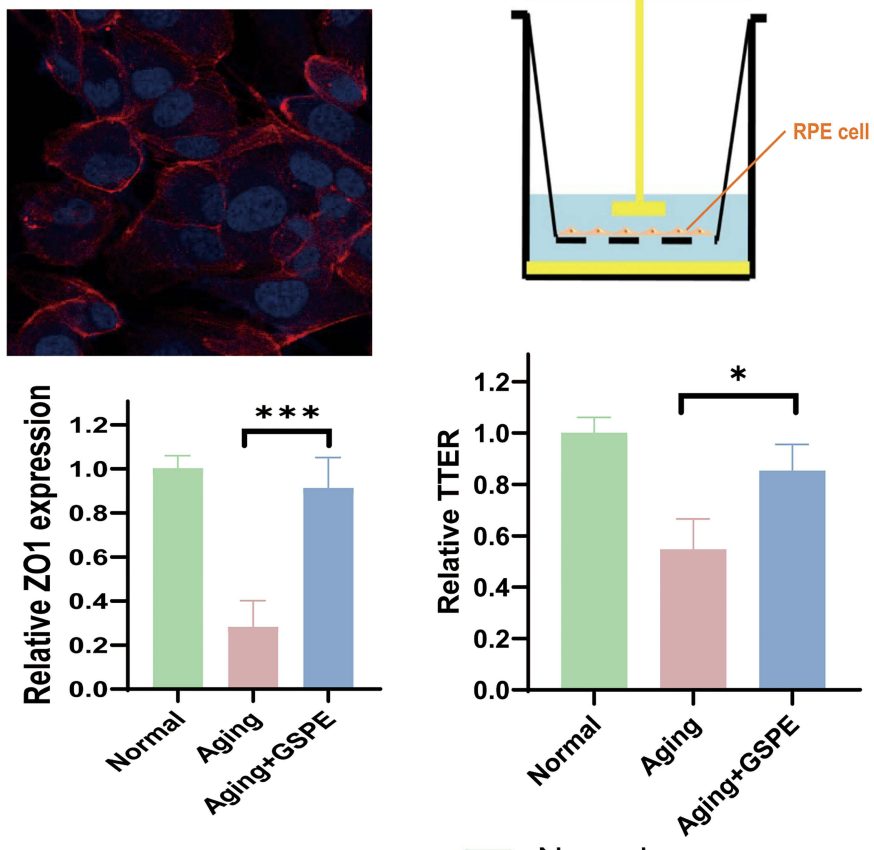

Normal

Aging

- Aging+GSPE RPE cell layer

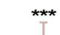

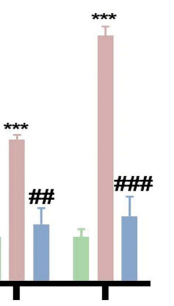

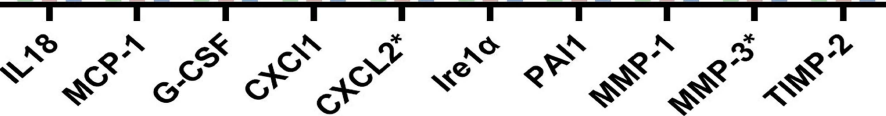

Figure 4 GSPE alleviated mitochondrial homeostasis, barrier function and SASP of aging RPE cells. (A) GSPE treatment significantly alleviated the mtDNA lesions, which was significantly increased in the aging RPE cells. $n=3$, ***: $P<0.001$. (B) After GSPE treatment, less monomer JI stained, and the permeability of the damaged mitochondrial membrane was significantly improved. The $\mathrm{JI}$ monomers were stained green and JI aggregates were stained red. $n=3, P<0.00 \mathrm{I}$. (C) GSPE could significantly improve the expression of ZOI in the aging RPE cells. ZOI was stained read and the nuclear was stained blue with DAPI. $n=3, P<0.001$. (D) GSPE treatment significantly improve the reinstate the TEER in the aging RPE cells. $n=3$, *: $P<0.05$. (E) Significantly expressed TGF- $\beta$ I, ILI $\beta$, IL6, IL8, MCP-I, G-CSF, CXCL2, MMP3 and TIMP-2 was detected and GSPE could significantly alleviate the expressions of most dysregulated SASP factors comparing with the aging RPE cells. *: the comparison of aging and normal groups. \#: the comparison of aging with GSPE treatment groups and aging groups. * and \#: $P<0.05$, ** and \#\#: $P<0.01$, *** and \#\#: $P<0.001$. 
A

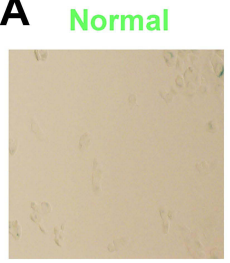

Aging+GSPE

+ Fk866

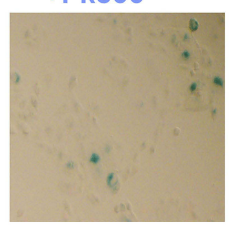

B

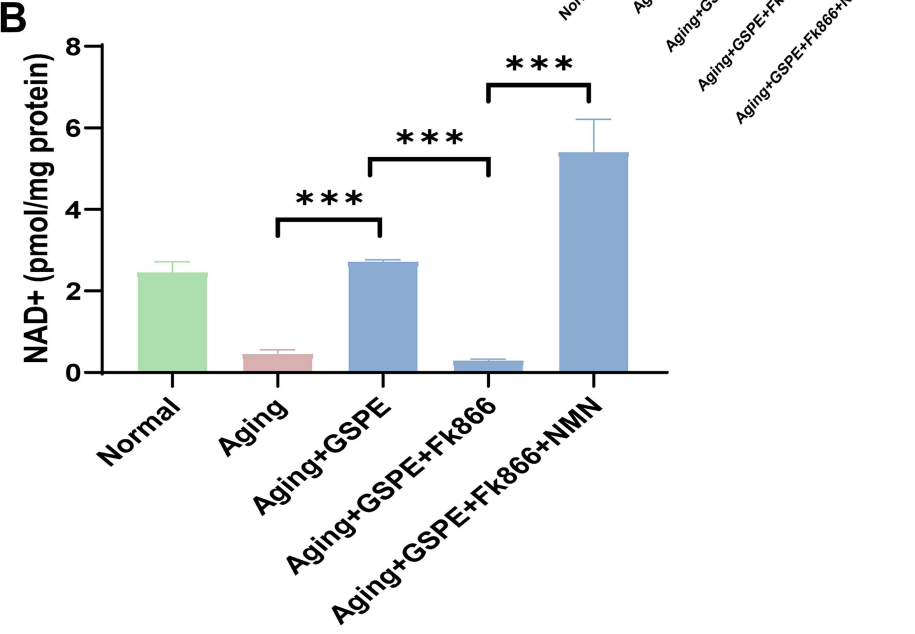

Aging+GSPE

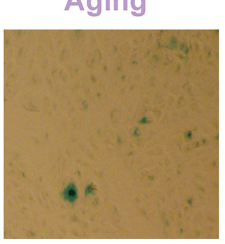

Aging+GSPE
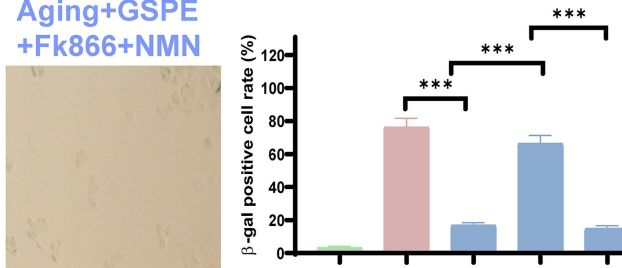
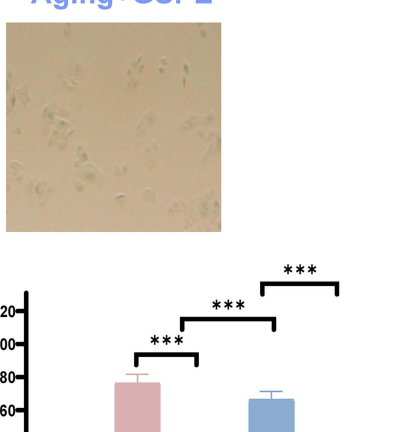

C

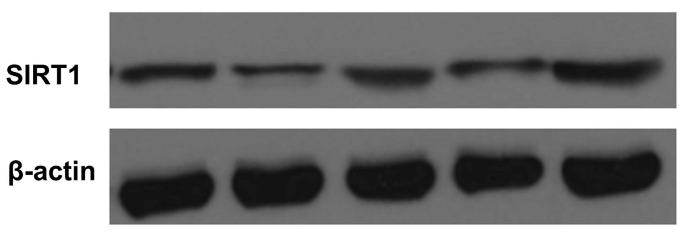

$\begin{array}{llllll}\text { Aging } & - & + & + & + & + \\ \text { GSEP } & - & - & + & + & + \\ \text { Fk866 } & - & - & - & + & + \\ \text { NMN } & - & - & - & - & +\end{array}$

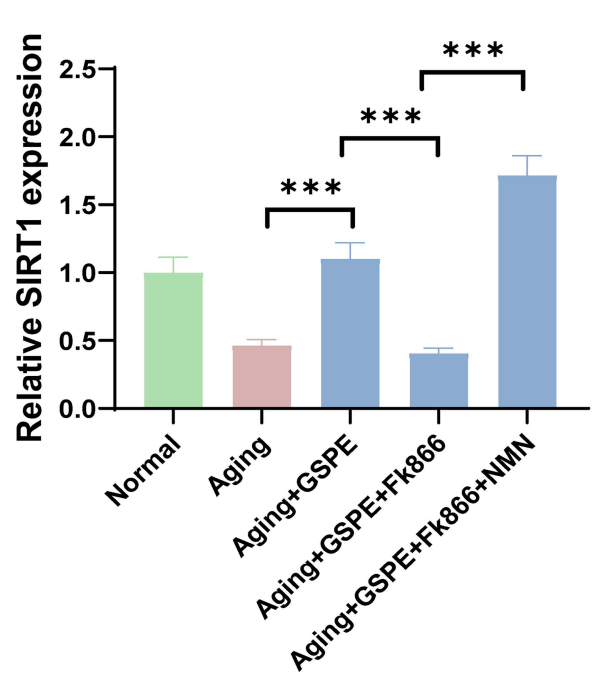

Figure 5 GSPE regulated NAMPT/SIRTI pathway in aging RPE cells. (A) The NAMPT inhibitor Fk866 blocked the protective effect of GSPE on senescent RPE cells, while supplementing with $\mathrm{NMN}$ significantly restored the protective effect. $\mathrm{n}=3, P<0.00 \mathrm{I}$. (B) GSPE demonstrated potential anti-aging effect through up-regulation of $\mathrm{NAD+}$ content. $n=3, P<0.001$. (C) GSPE treatment significantly up-regulated the expression of SIRTI and this regulation effects were modified by NAMPT inhibitor, Fk866, and $\mathrm{NAD+}$ precursor, NMN. The representative western-blot gels and statistical results were presented. $\mathrm{n}=3, * * *: P<0.001$.

that GSPE treatment demonstrated potential anti-aging effects through up-regulation of NAD+ contents (Figure 5B). As NAD+ was an important substrate of SIRT1, the effects of GSPE treatment on the NAMPT/ NAD+/SIRT1 pathway in aging RPE cells were detected. It was found that GSPE treatment significantly upregulated the expression of SIRT1 and this regulation effects were modified by NAMPT inhibitor, Fk866, and NAD+ precursor, NMN $(P<0.05$, Figure 5C).

\section{GSPE Alleviated the Inflammasome Activation Through SIRTI in Aging RPE Cells}

Inflammasome was associated with the incidence of various degenerative retinal disorders, the effects of GSPE on the inflammasome activity and the potential molecular mechanism was studied in this study. As showed in
Figure 6A, significantly increased NLRP3 expression was detected in the aging RPE cells and GSPE treatment reduced the NLRP3 expression after 24 or more hours treatment $(P<0.001)$. In the advanced experiments, 24 hours of GSPE treatment was used in the following mechanism detections. Since the regulatory effects of SIRT1 were reported in the previous experiments, the potential molecular mechanism of GSPE up-regulating SIRT1 and inhibiting inflammasome activity was studied. The treatment of GSPE significantly extenuated inflammasome activity and the key compounds, including NLRP3, ASC and caspase-1, were significantly alleviated by GSPE treatment in the aging RPE cells. While the suppressed NLRP3 pattern in the NMN treated aging RPE is impaired by SIRT1 inhibitor, EX-527 treatment and the detailed data are presented in Figure 6B. IL-1 $\beta$ and IL-18 were two cytokines related with the NLRP3 activation and it 

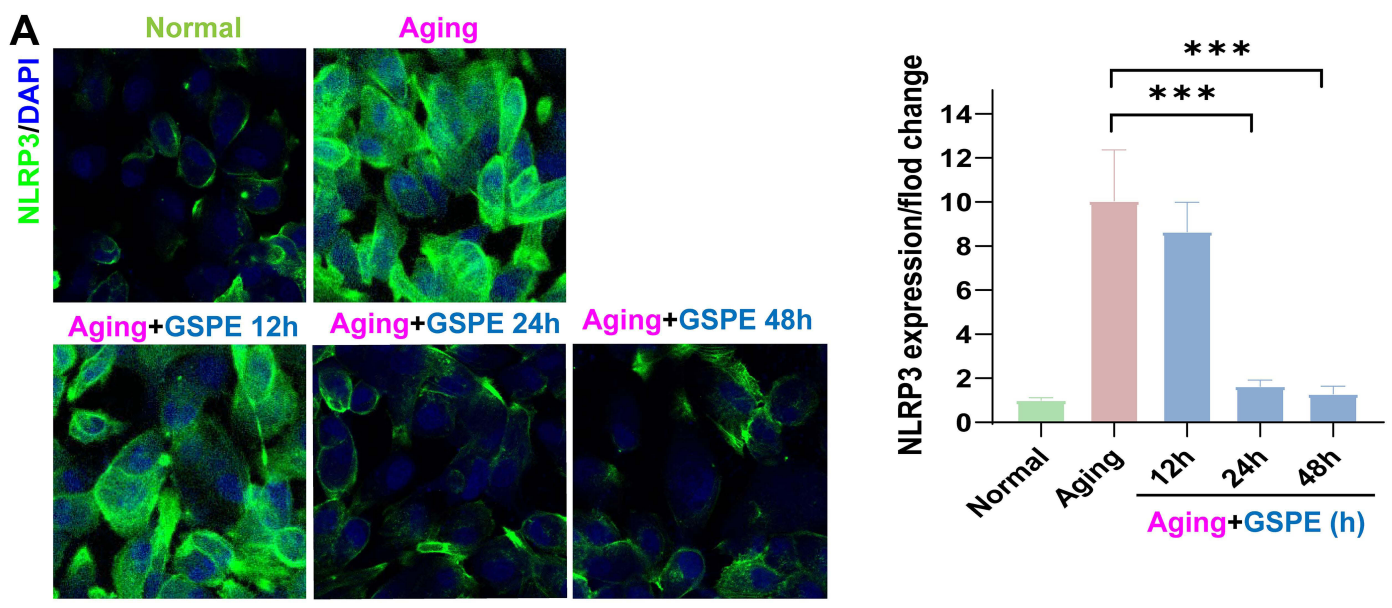

B

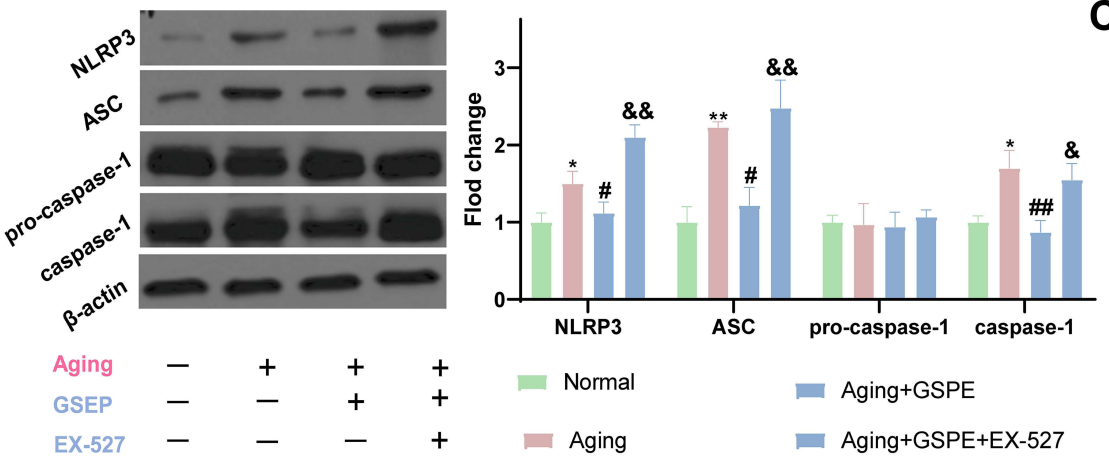

C

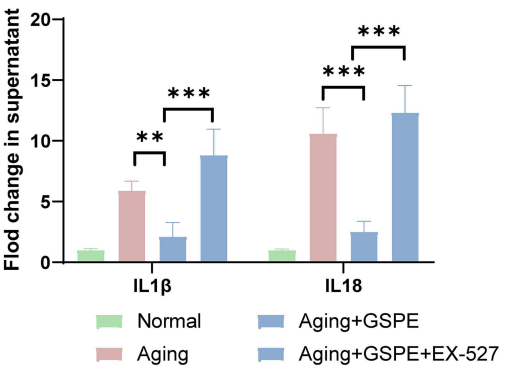

Figure 6 GSPE alleviated the inflammasome activation through SIRTI in aging RPE cells. (A) significantly increased NLRP3 was detected in the aging RPE cells and GSPE treatment reduced the NLRP3 expression after 24 or more hours treatment. The NLRP3 was stained greed and the nuclear was stained blue with DAPI. $\mathrm{n}=3, \mathrm{P}<0.00 \mathrm{I}$. (B) The treatment of GSPE significantly reduced the activity of inflammasomes such as NLRP3, ASC and caspase-I, and was significantly relieved in aging RPE cells. While SIRTI inhibited the NLRP3 regulation mode, it was processed with EX-527 and provided detailed data. The representative western-blot gels and statistical results were presented. *: the comparison of aging and normal groups. \#: the comparison of aging with GSPE treatment groups and aging groups, \&: The comparison of aging with GSPE+EX-527 treatment groups and aging with GSPE groups. $n=3, *$, \# and \&: $P<0.05$, **, \#\# and \&\&: $P<0.01$, ***, \#\# and \&\&\&: $P<0.001$. (C) The inhibitory effects of GSPE treatment on the ILI $\beta$ and IL-I8 secretions was disturbed by the SIRTI inhibitor, EX-527. $n=3, P<0.00$ I.

was found that the inhibitory effects of GSPE treatment on the IL1 $\beta$ and IL-18 secretions were disturbed by the SIRT1 inhibitor, EX-527 (Figure 6C).

\section{Discussion}

Cellular senescence was a major risk factor of the degenerative retinal disorders incidence. ${ }^{21}$ In this current study, GSPE consumption significantly increased the NAMPT expression and improved NAD+ contents in aging mice and thus alleviated the RPE cellular senescence. Aging would impair NAMPT function and lower NAD+ content was a cause of degenerative retinal diseases. In this study, advanced in-vitro studies demonstrated that GSPE could activate NAMPT expression and thus relieved $\mathrm{H}_{2} \mathrm{O}_{2}$ induced NAD+ depression. GSPE consumption could improve impaired mitochondrial functions in the aging RPE cells. By analyzing the effects of GSPE on SIRT1 and downstream inflammasome activity, it is demonstrated that GSPE demonstrates the protective effects in aging
RPE cells through NAMPT/SIRT1/NLRP3 pathway (Figure 7).

AMD was the most common irreversible cause of severe vision loss among the elderly in developed countries. Advanced age was one of the mostly accepted risk factors of the AMD; thus, cellular senescence might be associated with the incidence of AMD. ${ }^{22}$ The detailed mechanism of the cellular senescence in AMD progression remained unclear. The decline of NAMPT and its metabolic product, NAD+, was reported to be related to aging progress in different organs and cells, including RPE. ${ }^{22}$ Exogenous NAD+ precursor, NMN, demonstrated a significant protective role in RPE cellular senescence. ${ }^{23}$ NAMPT, which was the ratelimiting enzyme of NAD + metabolism and a potential target in the treatment of cellular senescence, might help to improve $\mathrm{NAD}+$ concentration and thus played an important role in the field of anti-aging. GSPE, which is a grape seed extract, is rich in polyphenolic bioflavonoid. In this current study, it was found that GSPE could significantly improve the NAMPT 


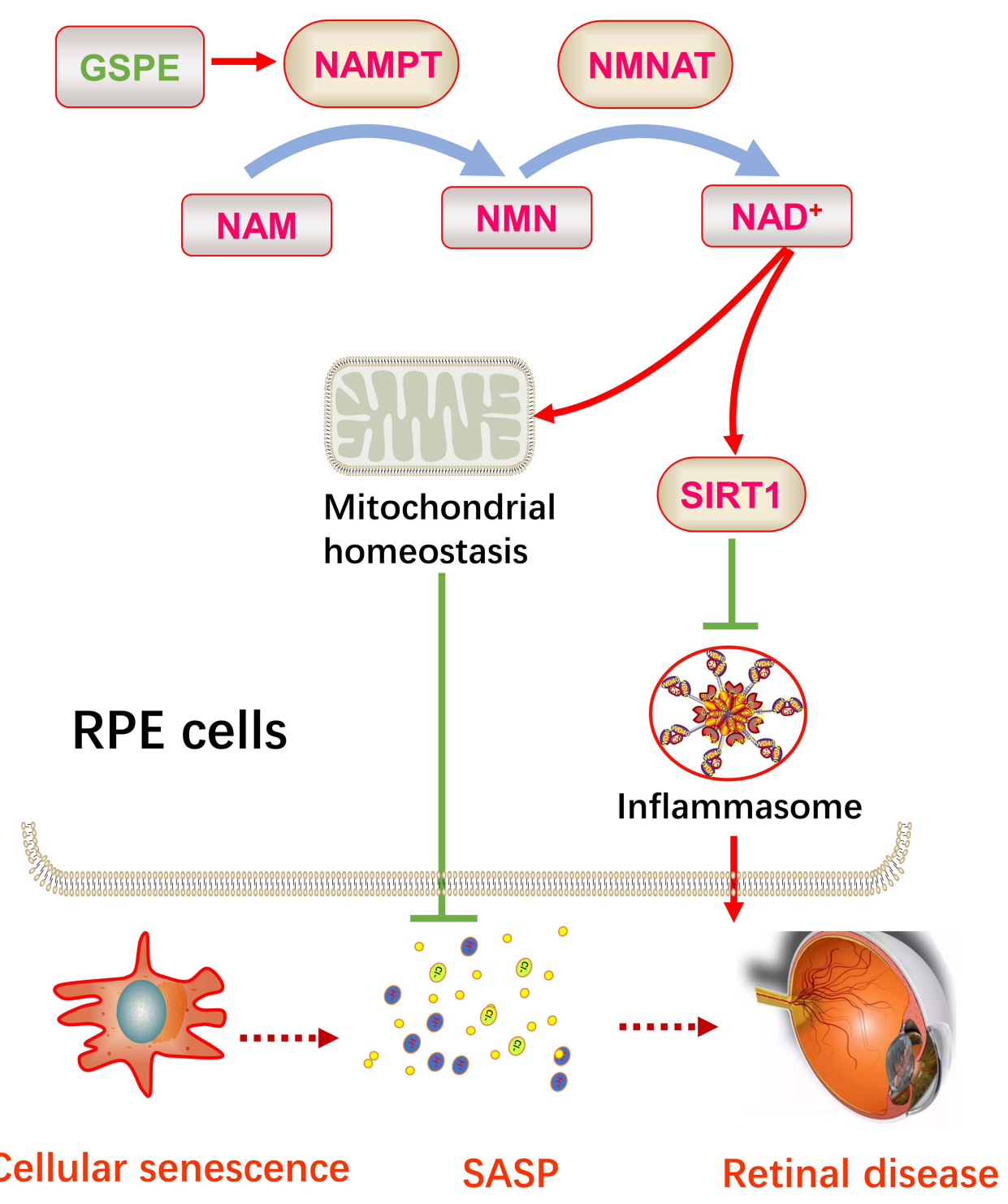

Figure 7 GSPE ameliorated cellular senescence in the RPE cells and could be a potential therapy for degenerative retinal diseases. GSPE significantly up-regulated the NAMPT expression in the aging RPE cells and thus improve the production of NAD+. Up-regulated NAD+ improved the expression of SIRTI and protected mitochondrial homeostasis, thus reducing SASP level. Improved cellular senescence status and lower SASP levels would be a retinal degenerative diseases.

expression as well as NAD+ content and thus protected the RPE cells from cellular senescence. NAD+ improvement had been regarded as an important approach; however, the most commonly used NAD+ supplement, NMN, was relatively expensive. Our study provided an economic NAD+ improvement therapy through up-regulation of the NAMPT expression in RPE tissues. The results of this study highlighted the importance of screening for more anti-aging natural extracts and would help to improve our understanding of their effects on aging-related degenerative disorders.

NAD + improvement could effectively improve various degenerative disorders; however, previous studies mainly focused on the effects of NAD + metabolism in retinal nerve cells, while less attention was paid to RPE cells. Considering that RPE senescence was a key process in various degenerative diseases, ${ }^{24}$ any treatment targeting RPE cellular senescence would provide huge effect on the management of these disorders. Rather than simply detecting the up-regulation of NAMPT and NAD+ by GSPE treatment, a more detailed molecular mechanism was studied. SIRT1 functioned based on the NAD+ activity and provided remarkable protections for retinal degenerative disorders. ${ }^{25,26}$ Based on the in-vitro experiments and molecular biological studies, it was found that GSPE application could significantly up-regulate the SIRT1 level. ${ }^{27}$ Inhibition of SIRT1 depleted the protective effects 
of GSPE significantly and it provided us the confirmation that SIRT1 was an important connection between GSPE treatment and the anti-aging effects in the RPE cells. As NAD + was a powerful activator of SIRT1, it was easy for us to understand the extract molecular mechanism for the protective effects of RPE cellular senescence: GSPE treatment significantly up-regulated the NAMPT levels in the aging RPE cells and thus improved the production of NAD + . Up-regulated NAD+ improved the expression of SIRT1, which was a key factor in the anti-aging function during NAD+ intervention. This study presented a simple but clear clue of the anti-aging effect of GSPE in RPE senescence; however, the complex NAD+ and SIRT1 regulation system required to be studied in advanced experiments. For instance, NAMPT could transform Nicotinamide (NAM) into NMN and NAMPT upregulation might lead to decreased NAM. NAM was an inhibitor of SIRT1 ${ }^{28}$ and NAM down-regulation might be associated with SIRT1 activator. More detailed experiments on the contributions of NAD + metabolism and SIRT1 activity to the RPE senescence were to be conducted.

Cellular senescence would lead to increased inflammatory cytokines expressions and this phenomenon was defined as SASP. $^{29,30}$ SASP was composed of a series of cytokines, including pro-inflammatory cytokines, growth factors, chemokines and matrix remodeling enzymes. The increased SASP could cause chronic low-grade inflammation and accelerate the aging progresses in both cells and organs. ${ }^{31,32}$ More and more evidence demonstrated that the chronic lowgrade inflammation was one of the most important manifestations of AMD. ${ }^{33}$ Although the precise mechanism had not been fully determined so far, we speculated that SASP was a link between aging and AMD. In this current study, the SASP markers were significantly increased in the aging mice and senescent RPE in-vitro models; thus, it provided evidence that SASP could be regarded as a key biological process for AMD development. Advanced experiments demonstrated that GSPE could reduce SASP status significantly. As SASP was both a hallmark and promoter of cellular senescence, GSPE could efficiently alleviate the retinal degenerative disorders by moderating SASP in aging senescence.

The physiological functions of RPE cells included growth factor secretion, blood-retinal barrier (BRB) maintenance and outer disc membrane phagocytosis. ${ }^{34}$ Pathological stress, such as aging, would lead to the loss of physiological functions and thus cause advanced pathological changes. Abnormal growth factor secretion of RPE was one of the most common pathological progresses and it would lead to neovascularization in choroidal (wet AMD) and retina (proliferative DR) ${ }^{35,36}$ RPE was the outer BRB and impaired RPE tight junction would cause retinal edema, such as macular edema secondary to DR. ${ }^{37}$ Both neovascularization and retinal edema were two important causes of blindness for patients with retinal disorders. In this current study, it was found that GSPE treatment could moderate the abnormal SASP levels as well as impaired BRB by alleviating RPE cellular senescence. Thus, GSPE supplement could be a potential treatment for the AMD and DR cases. More advanced experiments in diseased animal models and clinical trials in patients with the degenerative retinopathy would help us to understand the drug development potential of GSPE.

Based on the experimental data, two important cytokines, IL1 $\beta$ and IL8, were alleviated by GSPE treatment in both in-vivo and in-vitro aging models. Interestingly, these two cytokines were key effectors in the inflammasome activation. ${ }^{38,39}$ Inflammasome was a complex composed of multiple proteins, which could activate the protease caspase-1. Caspase-1 protease cleaved pro-IL $-1 \beta$ and pro-IL-18, activated them, and released active IL-1 $\beta$ and IL-18. As reported in previous studies, inflammasome was activated in degenerative retina, including AMD, DR and glaucoma. ${ }^{40,41}$ Previous studies on the effects of SIRT1 on the inflammation activation demonstrated that SIRT1 could moderate the inflammation activity significantly. Based on the results of a myocardial ischemia and reperfusion (I/R) mice model, SIRT1 agonist could reduce NLRP3 inflammasome activation during I/R. ${ }^{42}$ Combining the existing knowledge and experimental data in this study together, we could conclude that SIRT1 induced by GSPE was associated with the moderation of inflammasome activation during RPE cellular senescence. Considering that mounting evidence was reported on the relationship between inflammasome and cellular senescence in degenerative diseases, the findings in this study provided a new viewpoint on the crosstalk on the NAD+ metabolism and SIRT1/NLRP3 pathway in the RPE senescence.

This study had conducted the effects of GSPE in combination with NMN in RPE cellular senescence. An old age mouse animal model was used in this study, and it demonstrated the potential benefits of GSPE and NMN in retinal degeneration. Besides, we would conduct more advanced studies on the RPE cellular senescence-related disorders, including AMD, DR and RP. By analyzing the 
protective effects of NMN and GSPE treatments on other tissues, this would provide us with more knowledge.

In conclusion, this study has uncovered the protective effects of GSPE on the RPE senescence through upregulation of NAMPT expression and NAD+ concentration. NAD+ induced SIRT1 expression and then inhibited the activation of inflammasome complex during the process of RPE senescence. GPSE supplement provided a new potential therapeutic method in RPE senescence associated with retinal degenerative disorders.

\section{Acknowledgments}

This work was supported in whole or in part by the National Nature Science Foundation Project for Young Scientists of China (Grant No. 82000903, 81700804), the Foundation for Young Medical Talents of Jiangsu Province (Grant No. QNRC2016211), Scientific Research Project of Jiangsu Health Commission (Z2019044) and Youth Project of Henan Provincial Health and Health Commission, Ministry of Education (SB201902008).

\section{Disclosure}

Yan Wu is the founder of the Mosi Biotech Company. The authors report no other conflicts of interest in this work.

\section{References}

1. Blasiak J. Senescence in the pathogenesis of age-related macular degeneration. Cell Mol Life Sci. 2020;77(5):789-805.

2. Corbelli E, Parravano M, Sacconi R, et al. Prevalence and phenotypes of age-related macular degeneration in eyes with high myopia. Invest Ophthalmol Vis Sci. 2019;60(5):1394-1402.

3. Hyttinen JMT, Viiri J, Kaarniranta K, Blasiak J. Mitochondrial quality control in AMD: does mitophagy play a pivotal role? Cell Mol Life Sci. 2018;75(16):2991-3008.

4. Ueda K, Kim HJ, Zhao J, Song Y, Dunaief JL, Sparrow JR. Iron promotes oxidative cell death caused by bisretinoids of retina. Proc Natl Acad Sci U S A. 2018;115(19):4963-4968.

5. Yang X, Zhao L, Campos MM, et al. CSF1R blockade induces macrophage ablation and results in mouse choroidal vascular atrophy and RPE disorganization. Elife. 2020;9:e55564.

6. Wolk A, Upadhyay M, Ali M, et al. The retinal pigment epithelium in Sorsby Fundus Dystrophy shows increased sensitivity to oxidative stress-induced degeneration. Redox Biol. 2020;101681.

7. Amjad S, Nisar S, Bhat AA, et al. Role of $\mathrm{NAD}(+)$ in regulating cellular and metabolic signaling pathways. Mol Metab. 2021;49:101195

8. Chini CCS, Zeidler JD, Kashyap S, Warner G, Chini EN. Evolving concepts in NAD(+) metabolism. Cell Metab. 2021.

9. Wang Y, Grenell A, Zhong F, et al. Metabolic signature of the aging eye in mice. Neurobiol Aging. 2018;71:223-233.

10. Liebmann JM, Cioffi GA. Nicking glaucoma with nicotinamide? N Engl J Med. 2017;376(21):2079-2081.

11. Mekala NK, Kurdys J, Depuydt MM, Vazquez EJ, Rosca MG. Apoptosis inducing factor deficiency causes retinal photoreceptor degeneration. The protective role of the redox compound methylene blue. Redox Biol. 2019;20:107-117.
12. Jadeja RN, Powell FL, Jones MA, et al. Loss of NAMPT in aging retinal pigment epithelium reduces $\mathrm{NAD}(+)$ availability and promotes cellular senescence. Aging (Albany NY). 2018;10(6):1306-1323.

13. Cao G, Zeng X, Liu J, et al. Change of serum metabolome and cecal microflora in broiler chickens supplemented with grape seed extracts. Front Immunol. 2020;11:610934.

14. Wang L, Huang W, Zhan J. Grape seed proanthocyanidins induce autophagy and modulate survivin in HepG2 cells and inhibit xenograft tumor growth in vivo. Nutrients. 2019;11:12.

15. Ahmad SR, Gokulakrishnan P, Giriprasad R, Yatoo MA. Fruit-based natural antioxidants in meat and meat products: a review. Crit Rev Food Sci Nutr. 2015;55(11):1503-1513.

16. Nie Y, Sturzenbaum SR. Proanthocyanidins of natural origin: molecular mechanisms and implications for lipid disorder and aging-associated diseases. Adv Nutr. 2019;10(3):464-478.

17. Barbe A, Mellouk N, Rame C, et al. A grape seed extract maternal dietary supplementation improves egg quality and reduces ovarian steroidogenesis without affecting fertility parameters in reproductive hens. PLoS One. 2020;15(5):e0233169.

18. Chen C, Zhong Y, Wang JJ, et al. Regulation of Nrf2 by X box-binding protein 1 in retinal pigment epithelium. Front Genet. 2018;9:658.

19. Zhu W, Wu Y, Meng YF, et al. Effect of curcumin on aging retinal pigment epithelial cells. Drug Des Devel Ther. 2015;9:5337-5344.

20. Lin H, Xu H, Liang FQ, et al. Mitochondrial DNA damage and repair in RPE associated with aging and age-related macular degeneration. Invest Ophthalmol Vis Sci. 2011;52(6):3521-3529.

21. O'Mealey GB, Berry WL, Plafker SM. Sulforaphane is a Nrf2-independent inhibitor of mitochondrial fission. Redox Biol. 2017;11:103-110.

22. Marie M, Forster V, Fouquet S, et al. Phototoxic damage to cone photoreceptors can be independent of the visual pigment: the porphyrin hypothesis. Cell Death Dis. 2020;11(8):711.

23. Braidy N, Liu Y. NAD + therapy in age-related degenerative disorders: a benefit/risk analysis. Exp Gerontol. 2020;132:110831.

24. Wang S, Wang X, Cheng Y, et al. Autophagy dysfunction, cellular senescence, and abnormal immune-inflammatory responses in AMD: from mechanisms to therapeutic potential. Oxid Med Cell Longev. 2019;2019:3632169.

25. Miller JJ, Fink A, Banagis JA, et al. Sirtuin activation targets IDH-mutant tumors. Neuro Oncol. 2021;23(1):53-62.

26. Zhou Y, Wang S, Wan T, et al. Cyanidin-3-O-beta-glucoside inactivates NLRP3 inflammasome and alleviates alcoholic steatohepatitis via SirT1/NF-kappaB signaling pathway. Free Radic Biol Med. 2020.

27. Cai X, Bao L, Ren J, Li Y, Zhang Z. Grape seed procyanidin B2 protects podocytes from high glucose-induced mitochondrial dysfunction and apoptosis via the AMPK-SIRT1-PGC-1alpha axis in vitro. Food Funct. 2016;7(2):805-815.

28. Pan S, Leng J, Deng X, et al. Nicotinamide increases the sensitivity of chronic myeloid leukemia cells to doxorubicin via the inhibition of SIRT1. J Cell Biochem. 2020;121(1):574-586.

29. Coryell PR, Diekman BO, Loeser RF. Mechanisms and therapeutic implications of cellular senescence in osteoarthritis. Nat Rev Rheumatol. 2021;17(1):47-57.

30. Dominic A, Banerjee P, Hamilton DJ, Le NT, Abe JI. Timedependent replicative senescence vs. disturbed flow-induced pre-mature aging in atherosclerosis. Redox Biol. 2020;101614.

31. Guan Y, Zhang C, Lyu G, et al. Senescence-activated enhancer landscape orchestrates the senescence-associated secretory phenotype in murine fibroblasts. Nucleic Acids Res. 2020;48(19):10909-10923.

32. Borghesan M, Hoogaars WMH, Varela-Eirin M, Talma N, Demaria M, Senescence-Centric A. View of aging: implications for longevity and disease. Trends Cell Biol. 2020.

33. Faber C, Juel HB, Jensen BAH, et al. Chemokine expression in murine RPE/Choroid in response to systemic viral infection and elevated levels of circulating interferon-gamma. Invest Ophthalmol Vis Sci. 2019;60(1):192-201. 
34. Kaarniranta K, Pawlowska E, Szczepanska J, Blasiak J. DICER1 in the Pathogenesis of Age-related Macular Degeneration (AMD) - Alu RNA accumulation versus miRNA dysregulation. Aging Dis. 2020;11 (4):851-862.

35. Vega R, Carretero M, Bonilla LL. Anomalous angiogenesis in retina. Biomedicines. 2021;9(2):224.

36. Oubaha M, Miloudi K, Dejda A, et al. Senescence-associated secretory phenotype contributes to pathological angiogenesis in retinopathy. Sci Transl Med. 2016;8(362):362ra144.

37. Kuo C, Green CR, Rupenthal ID, Mugisho OO. Connexin 43 hemichannel block protects against retinal pigment epithelial cell barrier breakdown. Acta Diabetol. 2020;57(1):13-22.

38. Rao A, Strauss O, Kokkinou E, et al. Cytokines regulate the antigen-presenting characteristics of human circulating and tissue-resident intestinal ILCs. Nat Commun. 2020;11(1):2049.
39. Korhonen E, Bisevac J, Hyttinen JMT, et al. UV-B-induced inflammasome activation can be prevented by cis-urocanic acid in human corneal epithelial cells. Invest Ophthalmol Vis Sci. 2020;61(4):7.

40. Du J, Wang Y, Tu Y, et al. A prodrug of epigallocatechin-3-gallate alleviates high glucose-induced pro-angiogenic factor production by inhibiting the ROS/TXNIP/NLRP3 inflammasome axis in retinal Muller cells. Exp Eye Res. 2020;196:108065.

41. Mat Nor MN, Rupenthal ID, Green CR, Acosta ML. Connexin hemichannel block using orally delivered tonabersat improves outcomes in animal models of retinal disease. Neurotherapeutics. 2020;17(1):371-387.

42. Han Y, Sun W, Ren D, et al. SIRT1 agonism modulates cardiac NLRP3 inflammasome through pyruvate dehydrogenase during ischemia and reperfusion. Redox Biol. 2020;34:101538.

\section{Publish your work in this journal}

The Journal of Inflammation Research is an international, peerreviewed open-access journal that welcomes laboratory and clinical findings on the molecular basis, cell biology and pharmacology of inflammation including original research, reviews, symposium reports, hypothesis formation and commentaries on: acute/chronic inflammation; mediators of inflammation; cellular processes; molecular mechanisms; pharmacology and novel anti-inflammatory drugs; clinical conditions involving inflammation. The manuscript management system is completely online and includes a very quick and fair peerreview system. Visit http://www.dovepress.com/testimonials.php to read real quotes from published authors. 